\title{
An Economically Efficient Agent-based Autonomous Road Cleaner for Asian Cities
}

\author{
Ameer Hamza ${ }^{1}$, Faisal Riaz ${ }^{1}$, Samia Abid ${ }^{1 *}$, Gökhan Atalı ${ }^{2}$ and Khuram Nawaz Khayam ${ }^{1}$ \\ 0000-0002-3148-674X ${ }^{1}, 0000-0003-2005-3815^{2}, 0000-0003-1032-1724^{3}, 0000-0003-1215-9249^{4}, 0000-0002-9466-9412^{5}$
}

\begin{abstract}
${ }^{1}$ Control, Automotive and Robotics Lab affiliated lab of National Center of Robotics and Automation (NCRA HEC Pakistan), and with the Department of Computer Science and Information Technology, Mirpur University of Science and Technology (MUST), Mirpur-10250, AJK Pakistan ${ }^{4}$ Mechatronics Engineering, Sakarya University of Applied Sciences, Sakarya, 54050, Turkey
\end{abstract}

\section{Abstract}

In the last couple of decades, autonomous road cleaners have been enormously attracting the world. For this purpose, numerous researchers have contributed to designing efficient, robust, and human-friendly cleaning mechanisms. However, their proposed approaches do not provide a cost-effective solution due to the deployment of exorbitant sensors and sophisticated infrastructure deployment. Hence, we propose to develop a costeffective self-driving road that has the capability to clean the roads of major cities of Asia. The developed road cleaner efficiently detects road lanes using advanced machine vision techniques and estimates the angle to stay in the lane. Moreover, to control the speed of the road cleaner we have used a proportional-integral-derivative (PID) controller. The smart cleaning robot detects objects in its surroundings to prevent accidents in congested roads; thus it will have a function of anti-collision and automatic avoidance. It also measures turning angle by performing computations on the position of road lanes and takes turns accordingly. To validate the efficiency of the proposed solution, we have performed a series of experiments considering different test cases.

Keywords: Autonomous road cleaner; Human labor assistance; Robotics; Artificial Intelligence

\section{Research Article}

https://doi.org/10.30939/ijastech..997185

$\begin{array}{lr}\text { Received } & 18.09 .2021 \\ \text { Revised } & 09.11 .2021 \\ \text { Accepted } & 10.11 .2021\end{array}$

* Corresponding author

Samia Abid

samia.shah355@gmail.com

Address: NCRA HEC and with the Department of Computer Science and Information Technology, Mirpur University of Science and Technology (MUST), Mirpur-10250, AJK Pakistan

Tel: +923170303632

\section{Introduction}

The major cities of Asia are in dire need to be facilitated with advanced road cleaners. The dust, dirt, and other residuals on the road are a major problem for road occupants. The waste materials are harmful to the pedestrians as well as the vehicles. Hence to overcome the cleaning issues, certain waste management steps are required for a pollution-free environment.

The exponentially increasing population in Asian countries is an alarming situation that has laid pressure on urban utilities, services, and land [1]. To keep the environment clean, government officials have been opting for manual solutions by employing street sweepers for clean communities. A review has stated the occupational health hazards faced by the street sweepers and their vulnerability to bioaerosols [2]. Exposure to bioaerosol is related to throat, eyes, nose, and the infection of the respiratory system. Hence, it is required to take prudent countermeasures to replace the existing cleaning system with smart and efficient approaches.

The robotics manufacturers have developed numerous smart cleaners to clean dusted and polluted roads. For instance, FULONGMA has developed a road sweeping truck combining the ability of suction and cleaning [3]. It has been developed for cleaning municipal squares and city streets. In China, Idriverplus has developed an intelligent unmanned vehicle named 'Viggo'. Viggo has been designed to clean amusement parks, city streets, and factories [4]. Tromba Technologies startup has launched an autonomous street sweeper for paved areas [5]. Georgios Varisteas et al. [6] proposed to develop a low-cost self-driving vehicle using machine vision including a simple camera and Convolutional Neural Network $(\mathrm{CNN})$ which uses that vision for prediction and steering control. PID controller is implemented to control the throttle of the vehicle. Leonardo E et al. [7] proposed a solution for the smooth drive of the self-driving vehicle on the desired trajectory. Moreover, the PID controller is used for controlling the displacement of the vehicle. Mohamed Elhadi Hmidi et al. [8] introduced a dynamic model and PID controller for controlling the displacement and acceleration of the vehicle. Wael Farag [9] proposed an approach for the detection and tracking of Street lanes. Riaz et a. have proposed an enhanced emotion-enabled cognitive agent (EEEC_Agent) based controller for autonomous vehicles. It helps to avoid rear-end collisions with less number fuzzy rules, designed 
after fear emotion, and high efficiency [10].

However, the existing road cleaners cannot be considered complete road cleaning solutions while avoiding collisions with other road participants. According to an international survey, $70 \%$ of accidents occur due to minor mistakes of human drivers which cause major injuries [11]. In the literature, no such agent-based self-driving road cleaner has been proposed which is a true emulation of a human driver driving a common road cleaner. To overcome this problem, we have introduced a generic autonomous road cleaning framework and develop an autonomous road cleaner that is capable of driving from one point to another safely without any driver. The developed autonomous road cleaner framework consists of programmable autopilot, machine vision, object detection, and collision avoidance. Our proposed autonomous road cleaning framework could be used for road cleaning in public roads, industry, and amusement parks respectively. These autonomous road cleaners can be profitable for investors if they are deployed in their fields. According to the survey, people will prefer the self-driven road cleaner to the human-driven road cleaner because a self-driven road cleaner is much safer than a human-driven vehicle.

The rest of the paper is organized as follows. Section 2 provides a detailed discussion on the proposed mechanism of the smart road cleaner. Section 3 provides the results to validate the proposed methodology. While section 4 concludes the research work.

\section{Proposed Solution}

In this research work, we have developed a self-driving street cleaner that consists of different components including a high-definition camera, ultra-sonic SONAR, electric motors, edge computing devices, wheels' angle sensor, and brushes. We have detected road lines using advanced computer vision techniques. The agent architecture provided in the subsequent subsection represents the flow of this work.

\subsection{Proposed Agent Architecture}

The agent architecture of our proposed self-driving road cleaner is given in the figure below. This figure represents the flow of our proposed solution. In the sensory module, we have used ultrasonic SONAR and HD-camera to acquire input regarding the surroundings. The data acquired from ultrasonic SONAR is given to the object detection module which helps the cleaner to detect any static or dynamic objects on the road. While the HD camera provides visual data to the lane detection module to compute the angle between the detection lanes. The edge computing device transmits the control signal to the actuator module based on the data received from the object and lane detection modules. Based on these inputs, the actuator module of the self-driving cleaner activates the cleaning brushes and generates steering and throttle commands.

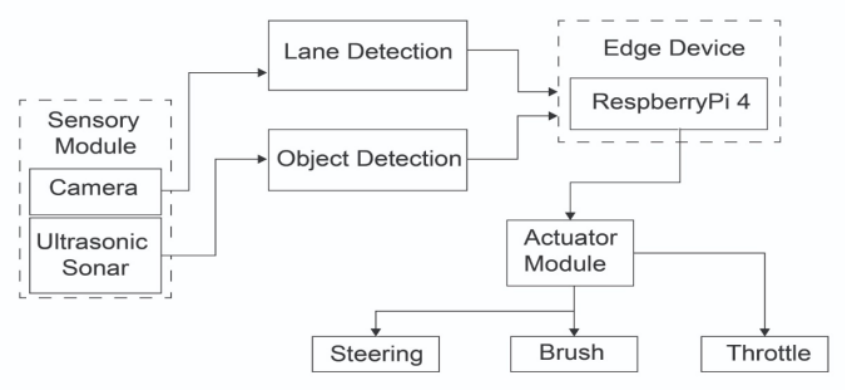

Fig. 1. The agent architecture of self-driving road cleaner

\subsection{Lane Detection}

The process of lane detection starts with the acquisition of an image from the HD camera at time ' $t$ '. From the acquired image, the region of interest (ROI) is selected by resizing it into specific pixels. In addition, the features are extracted from the respective image i.e., area, centroid, major and minor axis, etc. Based on the acquired features, the lanes are detected. We have used Canny for the detection of lane edges which helps us to detect the lanes using Hough Transform. The threshold is applied to make lane detection as smooth as possible. The objective of this process is to identify road lanes from the frames captured through the camera in realtime. From the detected lines, we draw a bases line connecting two parallel points on the lines to estimate the angle of navigation. Based on the baseline, a center point is estimated, and an imaginary line is drawn to estimate the angle between the baseline and the imaginary line. To compute the angle of navigation, we use Equation (1) as follows.

$$
x, y=\tan (\theta)=\frac{m 2-m 1}{1+m 2 * m 1}
$$

Equation (2) helps to compute the angle of deviation from the imaginary line to keep the vehicle in the center of the road lanes.

$$
\text { deviation }=\text { sta }-90
$$

While Equation (3) depicts that the angle of deviation will be set to zero if it lies between the mentioned bounds.

$$
\text { if }(-30<\text { deviation }<30) ; \text { deviation }=0
$$

By angle generated from lane detection and the threshold value, the edge computing device will send output signals to actuators (i.e. turn right, turn left). The front-wheel Angle sensor is utilized for keeping track of front tires. The rotation of the front wheels is controlled according to the input from the angle sensor. It will greatly reduce the risks of the motor getting damage occurred due to the full rotation of tires and still resisting to rotate. Moreover, it will also help in straightening the front tires after the turn is finished and a straight path is detected. 


\section{$\widehat{O M D=}$}

\subsection{PID Controller}

To control the speed of the road cleaner, we have used the PID controller. PID controller is used due to easy and fine-tuning of the parameters. We have tunes. PID controller by changing parameter gains to optimize the values. The equation to control speed employing a PID controller is given as follows.

$$
\text { speed }=(G p * P)+G i . I+G d . D
$$

where,

$$
\begin{aligned}
& P=e=a b s \text { (deviation) } \\
& I=\int_{0}^{t} e d t \\
& D=\frac{d(e)}{d t}
\end{aligned}
$$

While $\mathrm{P}$ represents the absolute steering angle, $I$ is the integration part of the controller obtained by taking the sum of the steering angle. $D$ is the derivation part of the controller obtained by finding the difference between the present steering angle with previous steering angle in Realtime.

\section{Results and Experiments}

In this section, we provide detailed discussions regarding the performed experiments and their results. The camera field of view obtained by the cleaner detected left \& right lanes and the suggested heading of the vehicle based on the steering angle is depicted in Figure 2.

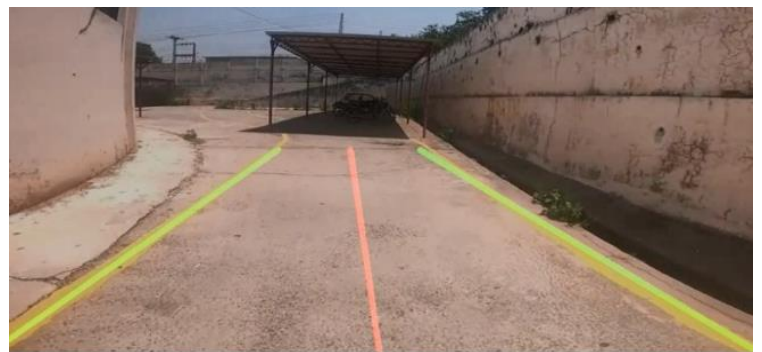

Fig. 2. Cleaner's field of view extracted lanes and heading as suggested by the algorithm

To validate the efficacy of the developed road cleaner, we have performed different in-field test cases.

\subsection{Test Case 1: Steering angle and speed control}

In the motion planning module, the autonomous road cleaner plans its path from origin to the destination point. The autonomous road cleaner performs steering control and speed control according to the input received from the camera in both straight and curved paths. Table 1 represents the results of steering and speed control and the accuracy achieved in different experiments.

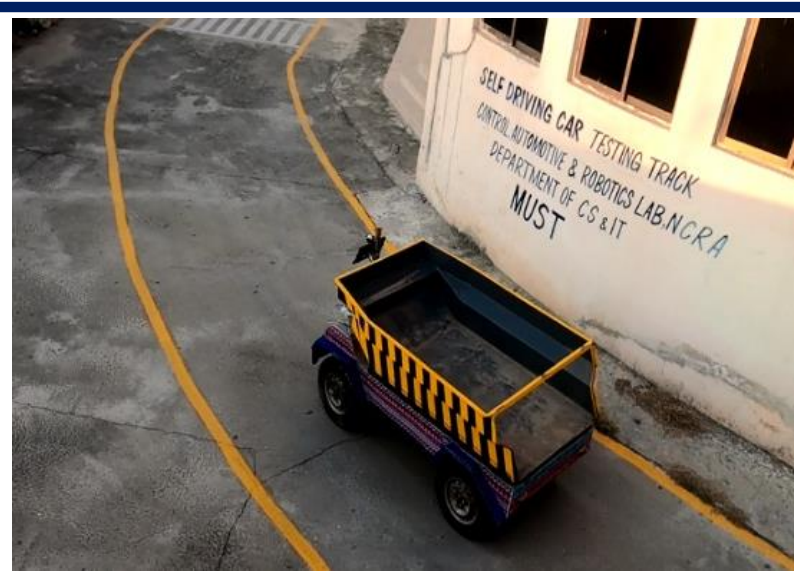

Fig 3. Steering control and speed control

Table 1. Experiment results of steering control and speed control

\begin{tabular}{c|c|c|c|c}
\hline No & $\begin{array}{c}\text { Turning } \\
\text { Angle }\end{array}$ & $\begin{array}{c}\text { Steering wheel } \\
\text { position }\end{array}$ & RPM & Accuracy \\
\hline 1 & 89 & Straight & 89.9962 & $98 \%$ \\
\hline 2 & 96 & Straight & 89.9962 & $97 \%$ \\
\hline 3 & 126 & Right & 82.8646 & $99 \%$ \\
\hline 4 & 98 & Straight & 87.1994 & $96 \%$ \\
\hline 5 & 123 & Right & 83.4553 & $99 \%$ \\
\hline 6 & 88 & Straight & 89.9858 & $97 \%$ \\
\hline 7 & 60 & Left & 84.0362 & $99 \%$ \\
\hline
\end{tabular}

\subsection{Test Case 2: Steering wheel angle sensor}

In this section, we have discussed test cases that are taken during the experiments of the angle sensor. By applying an algorithm and mechanism that is designed for the desired outcome from the $\mathrm{Au}-$ tonomous Streetcleaner. Table 2 represents the readings of steering wheel angle acquired as a result of different in-field experiments conducted.

Table 2. Experiment results of steering wheel angle sensor

\begin{tabular}{c|c|c|c}
\hline No & $\begin{array}{c}\text { Steering wheel } \\
\text { position }\end{array}$ & $\begin{array}{c}\text { Steering wheel } \\
\text { angle }\end{array}$ & Accuracy \\
\hline 1 & Straight & 710 to 790 & $98 \%$ \\
\hline 2 & Mild-Right & 791 to 1500 & $97 \%$ \\
\hline 3 & Full-Right & 1501 to 2400 & $99 \%$ \\
\hline 4 & Mild-Left & -100 to 709 & $96 \%$ \\
\hline 5 & Full-Left & -900 to -99 & $99 \%$ \\
\hline
\end{tabular}

\subsection{Test Case 3: Road Cleaning}

Here, we have provided test cases that are taken during the experiments of road cleaning. Figure 4 shows the testing of the road cleaner, sweeping the campus lanes. 
Table 3. Experiment results of road cleaning module

\begin{tabular}{c|c|c|c}
\hline No & $\begin{array}{c}\text { Sensor } \\
\text { Value }\end{array}$ & $\begin{array}{c}\text { Brushes Rota- } \\
\text { tion(r/m) }\end{array}$ & Accuracy \\
\hline 1 & 580 & 40 & $70 \%$ \\
\hline 2 & 585 & 36 & $77 \%$ \\
\hline 3 & 595 & 30 & $95 \%$ \\
\hline 4 & 600 & 23 & $80 \%$ \\
\hline 5 & 610 & 15 & $75 \%$ \\
\hline 6 & 615 & 10 & $60 \%$ \\
\hline
\end{tabular}

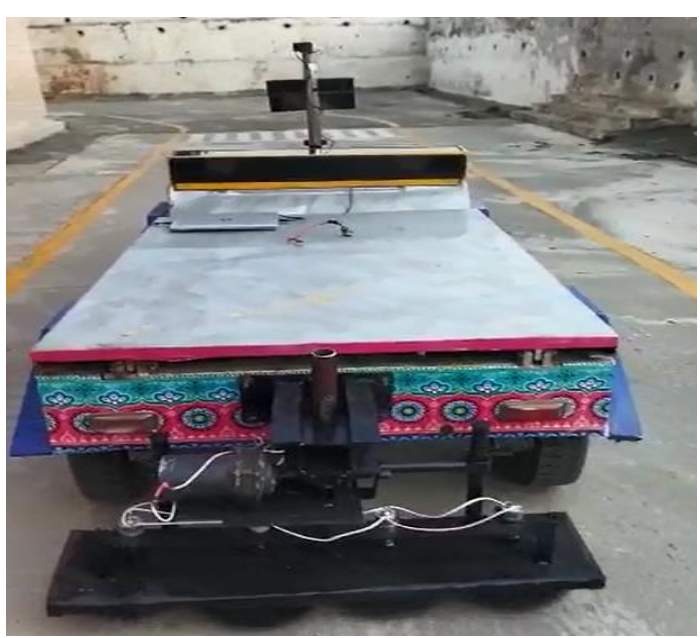

Fig. 4. The developed road cleaner

\section{Conclusions}

In this research work, a fully self-driving Road Cleaner is developed that is able to clean the roads of Asian cities. It will be costeffective as compared to other road cleaners available in the market. It is completely eco-friendly as it uses electricity as fuel from batteries. Electric motors are used as actuators of self-driving Road Cleaner. It is completely controlled by the edge computing device (i.e. Raspberry Pi 4). The edge computing device receives input from sensors such as the camera and ultrasonic sonars. The camera is used for machine vision recognition and ultrasonic sonar is used for object detection. The edge computing device applies different algorithms on the frames received for lane detection to calculate steering angle. The speed of the self-driving road cleaner is controlled by applying a PID controller. Moreover, the input received from ultrasonic sonars is used for anti-collision and automatic avoidance. By fusing all these features, the self-driving road cleaner can perform automated road cleaning of roads.

\section{Acknowledgment}

This study was supported by Control, Automotive, and Robotics (CAR) lab as researchers, we thank the National Centre of Robotics and Automation (NCRA), Higher Education and Commission (HEC), Islamabad, Pakistan.

\section{Nomenclature}

$I \quad:$ the proportional part

$P \quad:$ the integral part

$G_{p} \quad:$ the proportional gain, a tuning parameter

$G_{I} \quad:$ the integral gain, a tuning parameter

$G_{d} \quad:$ is the derivative gain, a tuning parameter

$e \quad$ : error

\section{Conflict of Interest Statement}

The authors declare that there is no conflict of interest in the study.

\section{CRediT Author Statement}

Ameer Hamza: Ideas, development and design of methodology, implementation of supporting algorithm

Faisal Riaz: Supervision, Methodology

Samia Abid: Writing-original draft, Review \& Editing

Gokhan Atalı: Review \& Editing, Visualization

Khuram Nawaz Khayam: Programming, testing of existing code components

\section{References}

[1] Van Kampen, Vera, Frank Hoffmeyer, Christoph Seifert, Thomas Brüning, and Jürgen Bünger. Occupational health hazards of street cleaners-a literature review considering prevention practices at the workplace. International Journal of Occupational Medicine and Environmental Health. 2020.

[2] Poole CJM, Basu S. Systematic Review: Occupational illness in the waste and recycling sector. Occup Med (Lond). 2017;67(8):626-36, https://doi.org/10.1093/occmed/kqx153.

[3] Fujian Longma Environmental Sanitation Equipment (2020) Highefficiency Street Sweeping Truck - FLM5186TSLDG6, Available at: https://www.fulongmagroup.com/high-efficiency-street-sweepingtruck-flm5186tsldg6/ (Accessed: 18th august 2021).

[4] Murray Slovick (Jul 27th, 2019) Autonomous Sweepers Keep Roads Clean in Major Chinese Cities, Available at: https://www.electronicdesign.com/markets/automotive/article/21808337/autonomous-sweepers-keep-roads-clean-in-major-chinese-cities (Accessed: 18th august 2021).

[5] Eugene Demaitre (September 29, 2020) Trombia Free autonomous street sweeper launched by Trombia Technologies, Available at: https://www.therobotreport.com/trombia-free-autonomous-streetsweeper-launched/ (Accessed: 18th august 2021).

[6] Varisteas, Georgios, Raphael Frank, Seyed Amin Sajadi Alamdari, Holger Voos, and Radu State. Evaluation of End-To-End Lear-ning for Autonomous Driving: The Good, the Bad and the Ugly. In 2019 2nd International Conference on Intelligent Autonomous Systems (ICoIAS), pp. 110-117. IEEE, 2019.

[7] Solaque G, Leonardo E., H. Guillermo Sanchez, and G. Adriana Riveros. Design and Test of a Path Tracking Controller to a High Capability Agricultural Vehicle. In Proceedings of the 5th International Conference on Mechatronics and Robotics Engineering, pp. 60-64. ACM, 2019. 
[8] Hmidi, Mohamed Elhedi, Ines Ben Salem, and Lilia El Amraoui. 2DOF PID for dynamic control of drive system hybrid vehicle electrical. In 2017 International Conference on Green Energy Conversion Systems (GECS), pp. 1-5. IEEE, 2017.

[9] Farag, W., \& Saleh, Z. Street Lane-Lines Detection in Real-Time for Advanced Driving Assistance Systems. 2018 Interna-tional Conference on Innovation and Intelligence for Informatics, Computing, and Technologies (3ICT). 2018. doi:10.1109/3ict.2018.8855797.
[10]Riaz, Faisal, and Muaz A. Niazi. Enhanced emotion enabled cognitive agent-based rear-end collision avoidance controller for autonomous vehicles. Simulation 94, no. 2018:(11); 957-977.

[11]Saleem, Hajira, Rehana Khatoon, Faisal Riaz, and Muhammad Atif Butt. Evaluating the role of neural networks and cyber security for the development of next generation autonomous vehicles: a survey. In Proceedings of the 4th International Electrical Engineering Conference. 2019. 